\title{
Rollen der Internetnutzer im Online-Diskurs am Beispiel der Interaktion in der Blogosphäre ${ }^{1}$
}

\author{
Roles of Internet Users in Online Discourse on Example of Interaction \\ in the Blogosphere
}

\begin{abstract}
ZUSAMMENFASSUNG
Zum Ziel des vorliegenden Beitrags wird die Bestimmung der Parameter zur Analyse von Rollen der Internetnutzer*innen im Online-Diskurs. Interaktionen in Neuen Medien sind rollengeprägt und die intentionalen multimodalen Handlungen der Online-Diskursteilnehmer*innen führen zur Entstehung von Konfigurationen, die bestimmte Interaktionsprofile etablieren (Pędzisz, 2017, S. 216-232). Der im Weiteren skizzierte theoretische Ansatz für ein allgemeines Modell der Beschreibung der Rollen von Blog-Interaktionsteilnehmer*innen soll die Mehrdimensionalität des rollengeprägten Profils der Blog-Interaktion rekonstruieren und nimmt den in der Blogosphäre realisierten Handlungstyp BLOGKOMMENTIEREN (Pędzisz, 2017, S. 73) in den Blick, der auf der Makro-Ebene des Online-Diskurses zum Handlungstyp ONLINE-KOMMENTIEREN wird. Es umfasst bestimmte Forschungsfragen zwecks der Darstellung von Blog-Interaktionsteilnehmer*innen als Funktionsträger, Individuen sowie Mitglieder der Sprach-, Kommunikations- und Diskursgemeinschaften (Adamzik, 2002) aufzuwerfen. Schlüsselwörter: Blogosphäre, Interaktionsprofil, Online-Diskurs, Blog-Interaktion
\end{abstract}

\section{ABSTRACT}

The aim of the article is to determine parameters for the analyzing the roles of internet users in online discourse. The interactions in new media are shaped by roles and the intentional multimodal actions of the online discourse participants, which create interaction profiles (Pędzisz, 2017, pp. 216-232). Based on the theoretical approach, the multidimensionality of the role-based profile of the blog interaction can be reconstructed. The focus is one type of action BLOGKOMMENTIEREN (p. 73), which becomes the type of action ONLINE-KOMMENTIEREN on the macro level of the online discourse. The model that describes roles of internet users in blog interactions includes research questions aimed at determining blog interaction participants, as function holders, individuals, and members of the languages, communication and discourse community (Adamzik, 2002).

Keywords: blogosphere, interaction profile, online discourse, blog-interaction

1 Die im Folgenden präsentierten Ausführungen ergänzen das in der Monographie Pędzisz (2017) vorgeschlagene Konzept des Interaktionsprofils um den breiteren Kontext des Online-Diskurses und gelten als die Aktualisierung des Forschungsstandes zum Handeln der Internetnutzer*innen in den Neuen Medien.

Joanna Pędzisz, Katedra Lingwistyki Stosowanej, Instytut Neofilologii, Uniwersytet Marii CurieSkłodowskiej, Pl. Marii Curie-Skłodowskiej 4a, 20-031 Lublin, joanna.pedzisz@wp.pl, https://orcid. org/0000-0002-0931-8387 


\section{Vorbemerkungen}

Der vorliegende Beitrag zielt darauf ab, Parameter zu der theoretischen Modellierung zu bestimmen, die eine Analyse von Rollen der Internetnutzer*innen im Online-Diskurs ermöglichen. Es wird davon ausgegangen, dass Interaktionen in Neuen Medien rollengeprägt sind und die intentionalen multimodalen Handlungen der Online-Diskursteilnehmer*innen zur Entstehung von Konfigurationen führen, die bestimmte Interaktionsprofile etablieren (Pędzisz, 2017, S. 216-232). Anhand der Analyse von Interaktionen in der Blogosphäre können Ziele, Intentionen und Interessen der Blog-Interaktionsteilnehmer*innen rekonstruiert und vor dem Hintergrund ihre Rollen definiert werden. Daraus resultiert die Tatsache, dass unter Rolle eine Konstellation von Handlungen der im Diskurs Handelnden verstanden wird, die abhängig vom Kommunikationsbereich, Medium, von Kommunikationsformen und produzierten Textsorten, von der Sprach- und Wissensbezogenheit der Handlungen sowie von Partizipationsmöglichkeiten der Handelnden etabliert wird. Da die BlogInteraktionen in dem Zusammenhang als hybridisierte Form der interpersonalen Online-Interaktionsprozesse auf der Mikro-Ebene und der gemeinschaftsbildenden Offline-Diskursprozesse auf der Makro-Ebene betrachtet werden (Pędzisz, 2013, S. 206), können die Rollen der Blog-Interaktionsteilnehmer*innen in dem Beitrag differenziert behandelt, um die Mehrdimensionalität des rollengeprägten Profils dieser Interaktion in den Vordergrund zu stellen. Demzufolge richtet sich der Fokus der hier präsentierten Ausführungen auf den in der Blogosphäre realisierten Handlungstyp BLOG-KOMMENTIEREN, der auf der Makro-Ebene des Online-Diskurses zum Handlungstyp ONLINE-KOMMENTIEREN wird. Im Anschluss an die textlinguistisch geprägte Definition der Handlung, nach der die Kommunizierenden mittels Texte handeln und als kommunikativ Handelnde „,mit ihren Intentionen, Erwartungen, Einstellungen zu Gegenständen und Sachverhalten/Ereignissen und vor allem zum jeweiligen Partner" (Heinemann \& Heinemann, 2002, S. 127) eine kommunikative Situation kognizieren, werden die Blog-Interaktionsteilnehmer*innen als Kommunizierende zugleich zu Diskursakteuren, derer multimodale Handlungen im Rahmen des ONLINE-KOMMENTIERENS in Anlehnung an Spitzmüller und Warnke (2011, S. 179) als Verfahren der Hörbarmachung von Positionen im OnlineDiskurs gelten und zwecks der Aushandlung des Autoritätsstatus realisiert werden.

Die im Beitrag formulierte Fragestellung gilt als theoretische Modellierung, anhand derer die Kategorien bestimmt werden, nach denen die Internetnutzer*innen als Blog-Interaktionsteilnehmer*innen und Diskursakteure (Spitzmüller \& Warnke, 2011) gekennzeichnet werden. Die Komplexität der Rollen von Internetnutzer*innen im Online-Diskurs wird dagegen dank der Darstellung der Blog-Interaktionsteilnehmer*innen als Funktionsträger, Individuen sowie Mitglieder der Sprach-, Kommunikations- und Diskursgemeinschaften (Adamzik, 2002) exponiert. 


\section{Zur Begrifflichkeit und Fragestellung}

Die in dem vorliegenden Beitrag präsentierten Ausführungen gehen auf die Parameter ein, nach denen die Spezifik einer intentionalen Handlungskonfiguration als Interaktionsprofils des in der Blogosphäre kreierten Online-Diskursausschnittes bestimmt werden kann.

Aus der sofware-technologischen Sicht gilt die Blogosphäre als Web 2.0-Angebot und -Anwendung. Demzufolge unterstützen die in der Blogosphäre möglichen Interaktivitätsformen wie Beitrags- und Feedbackinteraktivität (Bucher, 2013, S. 79) das interaktionale und intentionale Handeln der Blog-Interaktionsteilnehmer*innen, das mittels der Kommentare zum Blog oder Kommentare zu anderen Kommentaren vollzogen wird, denn, wie Antos (2019, S. 59) andeutet, u.a. Blogs ermöglichen zugängliche, schnelle, offene, komfortable und entgrenzende Interaktion und tragen zur Entstehung von Interaktions-Gemeinschaften bei. Daraus ergibt sich die Tatsache, dass das kommunikative Potenzial der Handlungen von BlogInteraktionsteilnehmer*innen und die von Bucher (2013, S. 80) postulierte kontinuierliche Sinnerzeugung in der Blog-Interaktion die „Architektur des Mitwirkens“ (Hinchcliffe, 2006, zit. nach Androutsopoulos, 2010, S. 421) ausmachen. Sie äußert sich hingegen in der medialen Vernetzung, die den wechselseitigen Austausch von Meinungen, Erfahrungen, Interessen, Informationen, Hobbys, sowie Werbung, politische Propaganda und die Präsentation von persönlichen oder organisatorischen Profilen fördert (Antos, 2019, S. 59). Mitwirken und Kontinuität als technologisch bedingte Phänomene in der Blogosphäre führen deswegen dazu, dass eine Konfiguration von Handlungen der Blog-Interaktionsteilnehmer*innen in Blog-Interaktionen entsteht (Pędzisz, 2017, S. 217). Da die miteinander konfigurierten Handlungstypen BLOG-KOMMENTIEREN und KOMMENTAR-KOMMENTIEREN als Interaktionsangebote und zugleich Diskursfragmente betrachtet werden können (S. 217), drängt sich die Frage auf, welche Mechanismen zur Etablierung eines Interaktionsprofils einer Blog-Interaktion als Online-Diskursausschnittes beitragen. Sowohl die Quantität als auch die Qualität der Bezüge auf einen Blog, einen Kommentar zum Blog oder einen Kommentar zum Kommentar, die von den Blog-Interaktionsteilnehmer*innen realisiert werden, gelten als Parameter, nach denen die Mechanismen eingehend erläutert werden können. Im Anschluss daran sollte sich der analytische Fokus erstens auf die Zahl a) der expliziten, b) der eindirektionalen Bezüge, c) der Interaktionsachsen d.h. der wechselseitigen Bezüge und d) der Bezüge ohne Reaktion (Pędzisz, 2017, S. 217) und zweitens auf die einzelnen Modi im Sinne der semiotischen Ressourcen wie Text, Grafik, Fotos, Design, Farbe, Layout, Tabellen etc. (Bucher, 2013, S. 64) richten, mit denen BlogInteraktionsteilnehmer*innen im Rahmen des BLOG- und KOMMENTAR-KOMMENTIERENS Bezug aufeinander nehmen.

Vor diesem Hintergrund kann eine methodologische Herangehensweise herausgearbeitet werden, nach der die multimodalen Handlungen der Blog- 
Interaktionsteilnehmer*innen in den Blick genommen werden, mit denen eindirektionale Bezüge und Interaktionsachsen in einer Blog-Interaktion aufgebaut werden. Nicht $\mathrm{zu}$ übersehen ist die Tatsache, dass anhand der multimodalen Handlungen Ziele, Intentionen und Interessen der Blog-Interaktionsteilnehmer*innen rekonstruiert werden können. Sie deuten ohne Zweifel auf Perspektiven, Ansichten und Meinungen hin, die die Blog-Interaktionsteilnehmer*innen im Interaktionsverlauf entwickeln und ihn dadurch profilieren.

\section{Rollen der Blog-Interaktionsteilnehmer*innen}

Außer Acht darf nicht gelassen werden, dass Rollen als Konstellationen von Handlungen der Blog-Interaktionsteilnehmer*innen konstruiert werden und die Etablierung von Interaktionsprofilen prägen. Das hat zur Folge, dass die quantitative und qualitative Analyse der Bezüge die Handlungen von BlogInteraktionsteilnehmer*innen fokussieren, um mit ihrer Hilfe die übernommenen Rollen zu rekonstruieren. Es wird davon ausgegangen, dass die Rollen erst im Laufe der Konstitution des Interaktionsprofils bestimmt werden. In Anlehnung an die von Adamzik (2002) vorgeschlagene Differenzierung der Rollen von Interaktanten lässt sich deswegen die Mehrdimensionalität des rollengeprägten Interaktionsprofils einer Blog-Interaktion als eines Online-Diskursausschnittes (S. 219) herausarbeiten. Unter Berücksichtigung der Voraussetzungen des interaktional linguistischen Ansatzes (Selting \& Couper-Kuhlen, 2000, S. 78-79) kann jedoch noch gezeigt werden, dass die sprachlichen (im Falle der BlogInteraktionsteilnehmer*innen: multimodalen) Handlungen rollenbedingt die Organisation einer Blog-Interaktion beeinflussen, als Manifestation bestimmter Interaktionspraktiken von Blog-Interaktionsteilnehmer*innen gelten und vor diesem Hintergrund strukturell und funktional organisiert sind. Gesonderte Erwähnung verdient noch die Interpretation der Interaktionspraktiken in der Blogosphäre. Sie werden als Praktiken gesehen, in denen das Soziale hervorgehoben wird. Schatzki (1996, S. 11) zufolge werden sie in dem im Weiteren konstruierten Modell als Bündel von Aktivitäten betrachtet, die typisiert, routinisiert, raumzeitlich-bindend und sozial verstehbar sind. Daraus folgt, dass die rollenbedingten multimodalen Handlungen die Blog-Interaktionsteilnehmer*innen als Subjekte darstellen, derer soziale Welt in einem routinisierten „nexus of doings and sayings“ (S. 89) zu identifizieren ist.

[S]ie verfügen über ein individuelles praktisches Verständnis, das ihren doings und sayings Sinn verleiht. Individuen sind in diesem Sinne zwar immer Teile der Netze aus Praktiken und Ordnungen, können aber als solche in der Regel auch autonom agieren, weil ihre doings und sayings nicht determiniert, sondern von den Praktik-Ordnung-Komplexen nur präfiguriert werden [Hervorhebung original] (Jonas, 2009, S. 18). 
Aus der Determiniertheit der multimodalen Handlungen von BlogInteraktionsteilnehmer*innen resultieren deswegen andere Rollen, die dank der in Blog-Interaktionen entstehenden Praktik-Ordnung-Komplexen etabliert werden.

\subsection{Blog-Interaktionsteilnehmer*innen als Akteure der illokutionären Rolle} Adamzik (2002, S. 230) weist darauf hin, dass die Beispielanalysen zur Kommunikation im öffentlichen Raum die Bestimmung einer illokutionären Rolle der Interaktanten entweder nicht vertiefen oder kaum in den Blick nehmen. An dieser Stelle muss aber die Tatsache hervorgehoben werden, dass eine illokutive Komponente der Handlungen von Interaktanten in unterschiedlichen Kommunikationsbereichen nicht minder wichtig ist. Sie ist auf die Intentionalität jeder menschlichen Handlung zurückzuführen und ihrer Realisierung liegen konkrete, von Heinemann und Heinemann (2002) hierarchisierte Ziele zugrunde, nämlich a) in einer bestimmten Situation antizipierte, auf die Veränderung von materiellen Objekten, sozialen Beziehungen oder emotiven Zuständen gerichtete fundamentale Ziele; b) Auxiliarziele, dank deren ein Partner in eine Interaktion einbezogen und beeinflusst wird; c) kollokutive Ziele, die über soziale Kontakte zu Partnern und über den Einsatz kommunikativer Mittel erreicht werden können; d) (Teil-)Ziele von einzelnen Sprachhandlungen (S. 19-20). Daraus kann geschlossen werden, dass Blog-Interaktionsteilnehmer*innen als Online-Diskussionsteilnehmer*innen in Neuen Medien gezielt, mit konkreten Absichten handeln und als Akteure betrachtet werden sollen, derer Rollen in einer illokutiven Handlung bestimmt werden. Im Anschluss daran drängen sich folgende Fragen auf:

1) Wie demonstrieren Blog-Interaktionsteilnehmer*innen ihre illokutive Rolle?

2) Ob und wie schreiben sie den anderen Blog-Interaktionsteilnehmer*innen eine illokutive Rolle zu?

\subsection{Blog-Interaktionsteilnehmer*innen als Funktionsträger}

Die Frage nach den von Blog-Interaktionsteilnehmer*innen repräsentierten Institutionen und Funktionen ist wegen der Spezifik der Neuen Medien schwer zu beantworten. Trotz der vertretenen Positionen und der übernommenen sozialen Rollen können Blog-Interaktionsteilnehmer*innen im Netz anonym bleiben. In dem Sinne gilt deswegen eine Blog-Interaktion als eine Form der öffentlichen, allgemein zugänglichen Kommunikation, in der ihre Akteure sozial relativ unbestimmt bleiben (Antos, 2019, S. 61). Blog-Interaktionsteilnehmer*innen müssen sich mittels der sprachlichen Bezeichnung konkreter Rolle nicht identifizieren. Es ist jedoch anzunehmen, dass sich ihre berufliche, institutionelle bzw. an eine bestimmte Ausbildung gebundene Rolle (Adamzik, 2002, S. 233) in einer Blog-Interaktion manifestiert und sie handeln im Netz nicht selten aus 
der Position der Vertreter von Institutionen, Instanzen oder als Funktionsträger. Somit bestätigt sich die These von Kaczmarek (2018), die im Anschluss daran auf diskursübergreifende Rollen verweist und ihr Wesen wie folgt begründet:

Diese Rollenmanifestationen gehen mit der Ausübung sozialer Funktionen, berufsbezogener Aufgaben und der Realisierung bereichsspezifischer Praktiken usw. einher, die den Zuständigkeits- bzw. Wirkungsbereich eines Medienakteurs widerspiegeln und nicht von der Spezifik eines jeweiligen Diskursausschnittes abhängen (S. 98).

Allerdings hat die Differenzierung von Adamzik (2002, S. 234) zwischen „Personen (innerhalb und) außerhalb einer bestimmten Kommunikationssphäre, zwischen Insidern und Außenstehenden“ zur Folge, dass der Grad der Involviertheit der Blog-Interaktionsteilnehmer*innen in einer Blog-Interaktion in den Blick genommen werden kann, womit die Konturen von Gruppen der Experten/ kompetenten Ansprechpartner und Laien/ des nicht fachkundigen Publikums, von Interessierten und Sachkundigen gezeichnet werden können. Rückt demnach die Sachkompetenz der Blog-Interaktionsteilnehmer*innen ins Blickfeld der Analyse von Interaktionsrollen in einer Blog-Interaktion als einem Online-Diskursausschnitt, sollen folgende Fragen aufgegriffen werden:
a. Mit welchen multimodalen Handlungen manifestieren Blog-Interaktion- steilnehmer*innen ihre Involviertheit in eine Blog-Interaktion?
b. Inwieweit sind Blog-Interaktionsteilnehmer*innen kompetent, um an einer Blog-Interaktion teilzunehmen?
c. Welche Dimensionen der Kompetenz sind in dem Zusammenhang zu nennen?
d. Wie demonstrieren Blog-Interaktionsteilnehmer den Grad ihrer Kompetenz?

\subsection{Blog-Interaktionsteilnehmer*innen als Diskursakteure}

Blog-Interaktionsteilnehmer*innen beziehen sich nicht selten auf umstrittene Fragen und äußern ihre Meinungen zu diesen Fragen. Angesichts dessen gelten sie als „privilegierte Akteure im gesellschaftlichen Diskurs“ (Adamzik, 2002, S. 234). Da sie sich zugleich an einem Online-Diskurs beteiligen, in dem auf seiner MakroEbene ein konkretes Thema oder Themen eines öffentlichen Mediendiskurses erörtert werden, sind Blog-Interaktionsteilnehmer*innen als Diskursakteure zu betrachten, derer Position im Diskurs als Anhänger, Befürworter, Gegner, Gegenredner, ablehnende und bejahende Gruppe bezeichnet wird (S. 234), und die sich dementsprechend diskursbedinge Rollen zuschreiben (Kaczmarek, 2018, S. 101). An dieser Stelle sei angemerkt, dass Spitzmüller und Warnke (2011) in ihrem Modell der diskurslinguistischen Mehr-Ebenen-Analyse DIMEAN die Kategorie der Diskursakteure als im Diskurs Handelnden einführen und ihre Funktion und Status definieren. 
Diskursakteure sind sowohl Individuen, Gruppen von Individuen, Netzwerke von Individuen, als auch nicht-personale Handlungsinstanzen wie Institutionen, Parteien, Medien usw., die zum Filter für die Zugehörigkeit von singulären Texten zu Diskursen werden (S. 172-173). Spitzmüller und Warnke (2011, S. 173) erklären das Wesen der Filterung von Aussagen folgendermaßen:

Eine Filterung erfolgt in zwei Richtungen: Diskurshandlungen filtern einerseits, welche Aussagen in einem Diskurs überhaupt eingehen. Welche Aussagen, welche Texte mit welchen Aussagen erscheinen überhaupt $[\ldots]$ ? Welche Positionen werden distribuiert, welche kommentiert, welche marginalisiert usw.? Entscheidend sind hier die >Diskursregeln Möglichkeitsbedingungen werden Aussagen hervorgebracht und eingeschränkt? [Hervorhebung original].

Es ist kaum zu übersehen, dass die Filterung von Aussagen, ihre Distribuierung, Kommentierung und Marginalisierung im engen Zusammenhang mit dem von Adamzik (2002, S. 234) thematisierten Grad der Involviertheit und mit den Kompetenzen stehen. Demzufolge zeigen sich Blog-Interaktionsteilnehmer*innen als Online-Diskursteilnehmer*innen. Sie machen Gebrauch von Web 2.0-Anwendungen auch gezielt und handeln in den im Netz präsenten Diskussionen angemessen und gemäß den (Interaktions)regeln.

Ohne Zweifel korrelieren auch das Konzept von „Voice- und Ideology Broker“ (Spitzmüller \& Warnke, 2011, S. 179-180) mit der von Adamzik (2002) gestellten Frage, „ob bzw. inwieweit jemand in einem bestimmten Sachbereich kompetent ist" (S. 234). Mit den Konzepten wird zum einen das Verfahren der Hörbar- und Unhörbarmachung von Positionen, zum anderen die Aushandlung der Autoritätsposition miteinbezogen, was konkrete Kompetenzen, Fähigkeiten sowie die von den im Diskurs Handelnden eingesetzten Strategien voraussetzt, nämlich die Fähigkeit, Aufmerksamkeit zu erreichen, den Positionen Gehör zu verschaffen, eigene Meinungen, Werte und Positionen bezüglich der diskutierten Themen durchzusetzen und Meinungen der anderen abzuschwächen (Spitzmüller \& Warnke, 2011, S. 179).

Außer Acht darf nicht gelassen werden, dass ein hoher Grad der Involviertheit der Blog-Interaktionsteilnehmer*innen darauf hinweist, dass sie ihren Autoritätsstatus aushandeln. Ihre Kommentare werden gelesen, bewertet und kommentiert. Demzufolge initiieren sie die Entstehung der Wechselbezüge, entwickeln Interaktionsachsen und positionieren sich diskursiv in einer Blog-Interaktion. Um verschiedene Facetten der Aushandlung von der Autoritätsposition zu beleuchten, muss die Tatsache hervorgehoben werden, dass nicht nur die Quantität der diskursiven Positionierungen sondern auch ihre Qualität relevant ist und auf den von Spitzmüller und Warnke (2011, S. 180) genannten metapragmatischen Äußerungen aufbaut: a) Autoritätenverweise; b) Betonungen eigener Expertise; c) sprachliche Bewertungsstrategien; d) implizite Positionierungsstrategien und 
e) Gate Keeping als Kontroll(versuch)en der Zugangsmöglichkeiten zum Diskurs nachgewiesen wird.

Hinsichtlich dessen lässt sich der Fragenkatalog zur Kennzeichnung der Blog-Interaktionsteilnehmer*innen als Autoritäten im Online-Diskursausschnitt ergänzen:

a. Mit welchen metapragmatischen Äußerungen lässt sich die qualitative Dominanz in einer Blog-Interaktion erreichen?

b. Wie werden die erkannten metapragmatischen Äußerungen in Anlehnung an die genannte Klassifikation von Spitzmüller und Warnke (2011, S. 180) gruppiert?

c. Mit welchen multimodalen Handlungen können Blog-Interaktionsteilnehmer den Ideology Broker-Status in einer Blog-Interaktion aushandeln?

\subsection{Individuen in einer Blog-Interaktion}

»Singularisierung«, also »das komplizierte Streben nach Einzigartigkeit« (Reckwitz 2017: 9), führt in sozialen Netzwerken paradoxerweise $\mathrm{zu}$ ausgesprochen kollektivistischen Moden, Lifestyle- und Lebensentwürfen, die in ihrer selfiehaften Selbstbezüglichkeit und Selbstzuwendung die Ideen einer >klassischen Öffentlichkeit paradoxerweise von sich weisen bzw. sie durch mediale Inszenierung zu kaschieren und ersetzen suchen [Hervorhebung original] (Antos, 2019, S. 63).

Im Anschluss daran drängt sich die Frage nach dem Status des Individuums und den Formen der Individualisierung in der öffentlichen Kommunikation auf, derer Realisierung eine Blog-Interaktion ohne Zweifel ist. In der differenzierten Betrachtung der Interaktionsrollen analysiert Adamzik (2002, S. 236-240) Relation: Privatperson - ihre Funktionsrollen - ihre Aufgaben in einer Interaktion. Die Forscherin hebt damit die Tatsache hervor, dass Interaktanten - Funktionsträger sowie Akteure im Diskurs - Subjekte sind, die das Individuelle in einer Interaktion zum Ausdruck bringen. Angesichts dessen rücken, Adamzik (2002) zufolge, vier Aspekte ins Zentrum der Aufmerksamkeit: 1) unterschiedliche Realisierung der Rolle des obligatorischen Akteurs; 2) Bereitwilligkeit, als Akteur typischer Rollen aufzutreten; 3) Bereitwilligkeit, als privilegierter Akteur (im Diskurs) aufzutreten; 4) Bereitwilligkeit, in der Öffentlichkeit als Privatperson aufzutreten.

Vor diesem Hintergrund greift Adamzik (2002) folgende Fragen auf:

- Inwieweit stellen Funktionsträger ihre Subjektivität in den Vordergrund?

- Inwieweit können Privatpersonen hinter ihren Funktionsrollen verschwinden und zu obligatorischen Akteuren werden?

- Wie lässt sich sprachlich individuelle Rollenperformanz markieren?

- Inwieweit sind Funktionsträger bereit, sich neben der Rolle als Funktionsträger anderen kommunikativen Aufgaben zu widmen, ,zu denen aber nicht nur sie Zugang haben und die sie teilweise auch gar nicht 
oder im Einzelfall nicht unbedingt realisieren müssen“" (S. 237)? Wie intensiv widmen sie sich dieser Aufgabe?

- Welche kommunikativen Aufgaben gelten als obligatorisch oder typisch? Welche kommunikativen Aufgaben sind untypisch oder verboten bzw. tabu?

- Welche und wie viele Personen sind aufgrund ihrer subjektiven Entscheidung bereit, sich an einem Diskurs zu beteiligen und seinen Verlauf zu beeinflussen?

- Entscheiden sich obligatorische Akteure infolgedessen für die Realisierung solcher kommunikativen Aufgaben, zu denen sie einen privilegierten Zugang haben, nicht ihren obligatorischen Aufgabenbereich ausmachen, „die sie aber freiwillig übernehmen“ (S. 239)?

Da es nur die Möglichkeit besteht, auf Blog, Kommentare zum Blog, Kommentare zu Kommentaren zu reagieren, wird die Rolle der Blog-Interaktionsteilnehmer*innen als obligatorischer Akteure ausgeschlossen. Der Status der Subjekte und die Identität der Blog-Interaktionsteilnehmer*innen werden hingegen anhand a) des Grades ihrer Involviertheit und ihrer Kompetenzen, die ermöglichen zwischen Experten und Laien, Interessierten und Sachkundigen zu unterscheiden, und b) des ausgehandelten Status Voice und Ideology Broker konstruiert. Hinsichtlich dessen gelten sie auch als Strategien zu Selbstdarstellungen, die nach Zielińska (2018) als Kommunikationsspiele verstanden werden können ${ }^{2}$. Infolge dessen gelten die analytischen Kategorien wie Grad der Involviertheit, Kompetenzgrad, VoiceStatus, Ideology Broker-Status ,als Bereiche, zu denen Blog-Interaktionsteilnehmer einen privilegierten Zugang haben, sie freiwillig übernehmen können, sie nicht realisieren müssen, was über den Umfang der typischen und untypischen bzw. Tabu-Aufgaben zwecks der Erreichung der Autoritätsposition oder des Status eines/ einer Sachkundigen entscheidet“" (Pędzisz, 2017, S. 225).

\subsection{Blog-Interaktionsteilnehmer*innen als Mitglieder der Sprach- und Kommunikationsgemeinschaft}

Es unterliegt keinem Zweifel, dass Blog-Interaktionsteilnehmer*innen als Mitglieder der Sprach- und Kommunikationsgemeinschaft (Adamzik, 2002, S. 240-242) zur Sprachgemeinschaft als Muttersprachler und zur Kommunikationsgemeinschaft Blog-Community gehören. An dieser Stelle sei jedoch angemerkt, dass die Verwendung von spezifischen Stilen, Registern, Sonder- und Fachsprachen durch eine konkrete Sprachgemeinschaft diese sprachlich als eine konkrete Community sichtbar macht und sozial konturiert (Antos, 2019, S. 58). Demnach steht die Interaktionsrolle von Blog-Interaktionsteilnehmer*innen als Mitgliedern einer Sprach- und

2 Mehr zum Konzept der Selbstdarstellung als Kommunikationsspiels in multimodalen Kommunikaten siehe Zielińska (2018, S. 295-312). 
Kommunikationsgemeinschaft mit der des Diskursakteurs im engen Zusammenhang. Aus den Handlungen von Diskursakteuren in einer Diskursgemeinschaft resultiert die „Vermarktung von Positionen“ (S. 58). Mittels Blogs, die neben Tweets, SMS, Mails etc. als computervermittelte Medienangebote betrachtet werden, werden individuelle und kollektive Wahrnehmungsprozesse, Formen der Gemeinschaftsbildung sowie die Konstitution digitaler Öffentlichkeit(en) verändert (vgl. S. 54). Obwohl Hahn, Hohlfeld und Knieper (2017) die traditionellen Konzepte der Öffentlichkeit in der computervermittelten Kommunikation in Frage stellen ${ }^{3}$, weisen BlogInteraktionsteilnehmer*innen als Blog-Community bestimmte kommunikative Kompetenzen nach, die ermöglichen, eine Blog-Interaktion zwecks der Konstituierung und Stabilisierung einer Gemeinschaft (Antos, 2019, S. 54) zu etablieren und zu führen. Die dadurch mittels konkreter multimodaler Handlungen entwickelte Art der „InsiderKommunikation" (Adamzik, 2002, S. 243) gilt als ein Beweis für eine Vertrautheit der Blog-Interaktionsteilnehmer*innen mit dem Kommunikationsbereich Blogosphä$r e$ und den Regeln seines Funktionierens auf der Ebene der Organisation der Interaktion. In dem Sinne wird eine Blog-Community mit ihren erfahrenen Teilnehmern (ebenda) zu einer Gemeinschaft, die eine Handlungskonfiguration als Interaktionsprofil konstruiert. Im Anschluss daran entscheiden der Grad der Vertrautheit der BlogCommunity mit der interaktionalen Organisation der Blogosphäre und der Umfang der (sprachlich-kommunikativen) Kompetenz der Blog-Interaktionsteilnehmer*innen über den Status eines erfahrenen Teilnehmers, woraus sich zugleich die Differenzierung zwischen Experten/erfahrenen Teilnehmern und Laien/Neulingen ergibt.

Die von Spitzmüller und Warnke (2011, S. 182) definierten Kommunikationsbereiche ermöglichen dagegen nach Habscheid und Fix (2003) die Analyse von Formen der sprachlichen Inszenierung sozialer Zugehörigkeiten in behandelten Kommunikationsbereichen. Deswegen ,wird die Blogosphäre als Kommunikationsbereich betrachtet, in dem Blog-Interaktionsteilnehmer als Blog-Community mittels der multimodalen Handlungen ihre Rollen als Insider/erfahrene Teilnehmer/Experten und Außenstehende/Neulinge/Laien manifestieren“ (Pędzisz, 2017, S. 226). Damit geht einher, dass das Recht auf (aktive) Teilnahme an einer BlogInteraktion (Adamzik, 2002, S. 243) mit der folgenden Fragestellung verifiziert werden kann:

a. Mit welchen multimodalen Handlungen vollzieht sich die Manifestierung der Zugehörigkeit von Blog-Interaktionsteilnehmer*innen als erfahrenen Teilnehmer*innen zur Blog-Community?

3 Das Internet mitsamt seinen Formen computervermittelter Kommunikation hat zu einer Fragmentierung der Öffentlichkeit geführt. Als Tatsache ist dies unumstritten, über die Folgen der digitalen Teil- und Nischenöffentlichkeiten etwa für die gesellschaftliche Integration herrscht dagegen Uneinigkeit. Die Frage, ob solche ,persönlichen Öffentlichkeiten' sozialer Netzwerke zur Atomisierung des Publikums und zum Leben in einer Filter Bubble sensu Eli Pariser führen, wird derzeit unterschiedlich beantwortet (Hahn, Hohlfeld, \& Knieper, 2015, S. 11). 
b. Mit welchen multimodalen Handlungen identifizieren Blog-Interaktionsteilnehmer*innen als Insider den Grad der Vertrautheit mit der BlogCommunity und mit den Interaktionsregeln in der Blogosphäre?

c. Mit welchen multimodalen Handlungen beschränken Blog-Interaktionsteilnehmer*innen das Recht auf aktive Teilnahme an einer Blog-Interaktion?

d. Mit welchen multimodalen Handlungen räumen Blog-Interaktionsteilnehmer*innen das Recht ein, an einer Blog-Interaktion teilzunehmen?

Die Fragen greifen auf Gate Keeping als Kontroll(versuch)e der Zugangsmöglichkeiten zum Online-Diskurs und die dadurch ausgehandelte Autoritätsposition in einer Blog-Interaktion als einem Online-Diskursausschnitt auf.

\section{Ausblick}

Die in dem Beitrag genannten Parameter zur Modellierung und Analyse von Rollen der Internetnutzer*innen im Online-Diskurs fokussieren eine konkrete Form der Realisierung eines Online-Diskurses als öffentlicher Kommunikation, nämlich eine Blog-Interaktion. Die hier präsentierte Differenzierung der Rollen, die BlogInteraktionsteilnehmer*innen übernehmen, nimmt allgemein zugängliche Bezeichnungen wie Fan, Follower, Beobachter, Kritiker, Befürworter, wir, ich, Sie, Publikum in den Blick und deutet darauf hin, wie Blog-Interaktionsteilnehmer*innen als Gruppen, Individuen, Diskursakteure in einer Blog-Community sprachlich etabliert werden. Der Beitrag sollte deswegen zeigen, dass der von Bock und Antos (2019) postulierte, durch digitale Revolution geprägte Strukturwandel der Öffentlichkeit und der Rollen von Laien und Experten zwar legitim ist, aber die hier präsentierte methodologische Herangehensweise konturiert die Antwortperspektive der von Hauser, Opiłowski und Wyss (2019) aufgeworfenen Frage: „Was machen die Menschen mit den sozialen Medien und was machen die sozialen Medien mit den Menschen?" (S. 8). Somit geht das Konzept des rollengeprägten Interaktionsprofils auf die Ansätze ein, die die Entsubjektivierung der Diskurse hinterfragen (u.a. Albert, 2008; Angermüller, 2008; Czachur, 2011; Heinemann, 2011; Kaczmarek, 2018), und versucht die These über die Fragmentierung, Marginalisierung und Atomisierung der Öffentlichkeit (Hahn, Hohlfeld \& Knieper, 2017, S. 11) zu verifizieren.

\section{References}

Adamzik, K. (2002). Interaktionsrollen. Die Textwelt und ihre Akteure. In K. Adamzik (Ed.), Texte, Diskurse, Interaktionsrollen. Analysen zur Kommunikation im öffentlichen Raum (pp. 211-255). Tübingen: Stauffenburg.

Albert, G. (2008). Die Konstruktion des Subjektes in Philosophie und Diskurslinguistik. In I. H. Warnke, \& J. Spitzmüller (Eds.), Methoden der Diskurslinguistik. Sprachwissenschaftliche Zugänge zur transtextuellen Ebene (pp. 151-182). Berlin, New York: De Gruyter.

Angermüller, J. (2008). Wer spricht? Die Aussagenanalyse am Beispiel des Rassismus-Diskurses. In I. H. Warnke, \& J. Spitzmüller(Eds.), Methoden der Diskurslinguistik. Sprachwissenschaftliche Zugänge zur transtextuellen (pp. 185-206). Berlin, New York: De Gruyter. 
Antos, G. (2019). Medien, Wahrnehmung, Öffentlichkeit. Wahrnehmungs-Gemeinschaften und deren Interaktion als Gegenstand der Medienlinguistik. In S. Hauser, R. Opiłowski, \& E. L. Wyss (Eds.), Alternative Öffentlichkeiten. Soziale Medien zwischen Partizipation, Sharing, Vergemeinschaftung (pp. 53-80). Bielefeld: transcript.

Bock, B. M., \& Antos, G. (2019). „Öffentlichkeit“-, „Laien“- „Experten“: Strukturwandel von „Laien“ und „Experten“ in Diskursen über „Sprache“. In G. Antos, T. Niehr, \& J. Spitzmüller (Eds.), Handbuch Sprache im Urteil der Öffentlichkeit (pp. 54-80). Berlin, New York: De Gruyter.

Bucher, H.-J. (2013). Online-Diskurse als multimodale Netzwerk-Kommunikation. Plädoyer für eine Paradigmenerweiterung. In C. Fraas, S. Meier, \& Ch. Pentzold (Eds.), Online-Diskurse. Theorien und Methoden transmedialer Online-Diskursforschung (pp. 57-101). Köln: Herbert von Halem Verlag.

Czachur, W. (2011). Diskursive Weltbilder im Kontrast. Linguistische Konzeption und Methode der kontrastiven Diskursanalyse deutscher und polnischer Medien. Wrocław: ATUT.

Habscheid, S., \& Fix, U. (Eds.). (2003). Gruppenstille. Zur sprachlichen Inszenierung sozialer Zugehörigkeit. Frankfurt: Peter Lang Verlag.

Hahn, O., Hohlfeld, R., \& Knieper, T. (2015). Einführung: Digitale Öffentlichkeit - Digitale Öffentlichkeiten. In O. Hahn, R. Hohlfeld, \& T. Knieper (Eds.), Digitale Öffentlicheit(en) (pp. 11-18). Köln: Herbert von Halem Verlag.

Hauser, S., Opiłowski, R., \& E. L. Wyss (2019). Alternative Öffentlichkeiten in sozialen Medien - einleitende Anmerkungen. In S. Hauser, R. Opiłowski, \& E. L. Wyss (Eds.), Alternative Öffentlichkeiten. Soziale Medien zwischen Partizipation, Sharing, Vergemeinschaftung (pp. 7-12). Bielefeld: transcript.

Heinemann, W. (2011). Diskursanalyse in der Kontroverse. tekst $i$ dyskurs - text und diskurs, 4, 31-67.

Heinemann, M., \& Heinemann, W. (2002). Grundlagen der Textlinguistik. Interaktion - Text Diskurs. Tübingen: Niemeyer.

Jonas, M. (2009). The social site approach versus the approach to discourse/practice formations. Reihe Soziologie, 92. Retrieved January 1, 2021, from http:/www.ihs.ac.at/publications/soc/ rs92.pdf.

Kaczmarek, D. (2018). Binationale Diskursanalyse. Grundlagen und Fallstudien zum deutschpolnischen medialen Gegendiskurs. Łódź: Wydawnictwo Uniwersytetu Łódzkiego.

Pędzisz, J. (2013). Diskursive Blog-Interaktion: massenmediale oder interpersonale Diskursrealisation? tekst $i$ dyskurs - text und diskurs, 6, 203-217.

Pędzisz, J. (2017). Profil des Online-Diskurses in Blog-Interaktionen an der Schnittstelle zwischen theoretischem Konzept und empirischem Modell. Frankfurt am Main: Peter Lang Verlag.

Reckwitz, A. (2017). Die Gesellschaft der Singularitäten. Zum Strukturwandel der Moderne. Berlin: Suhrkamp.

Schatzki, T. R. (1996). Social Practices. A Wittgensteinian approach to human activity and the social Cambridge: Cambridge University Press.

Selting, M., \& Couper-Kuhlen, E. (2000). Argumente für die Entwicklung einer „interaktionalen“ Linguistik. Gesprächsforschung - Online-Zeitschrift zur verbalen Interaktion, 1, 76-95.

Spitzmüller, J., \& Warnke, I. H. (2011). Diskurslinguistik. Eine Einführung in Theorien und Methoden der transtextuellen Sprachanalyse. Berlin, Boston: De Gruyter.

Zielińska, K. (2018). Multimodale Selbstdarstellungen und die Strategien der Imagepflege in multimodalen Kommunikaten. Methodische Überlegungen. tekst i dyskurs - text und diskurs, 11, 295-312. DOI: 10.7311/tid.11.2018.12. 